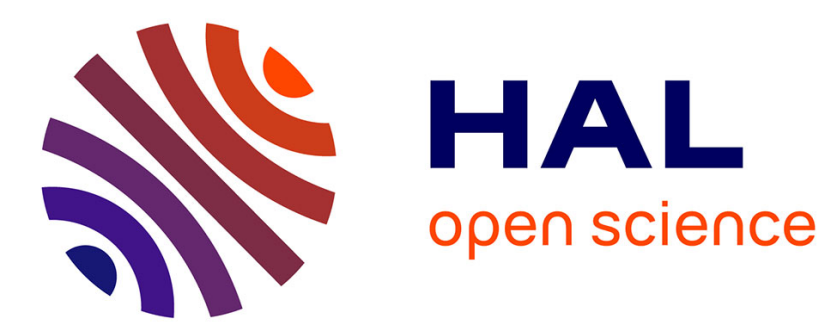

\title{
VUV spectroscopic investigation of HTSC and C60 materials
}

\author{
V. Stankevitch
}

\section{To cite this version:}

V. Stankevitch. VUV spectroscopic investigation of HTSC and C60 materials. Journal de Physique IV Proceedings, 1994, 04 (C9), pp.C9-83-C9-88. 10.1051/jp4:1994910 . jpa-00253470

\section{HAL Id: jpa-00253470 https://hal.science/jpa-00253470}

Submitted on 1 Jan 1994

HAL is a multi-disciplinary open access archive for the deposit and dissemination of scientific research documents, whether they are published or not. The documents may come from teaching and research institutions in France or abroad, or from public or private research centers.
L'archive ouverte pluridisciplinaire HAL, est destinée au dépôt et à la diffusion de documents scientifiques de niveau recherche, publiés ou non, émanant des établissements d'enseignement et de recherche français ou étrangers, des laboratoires publics ou privés. 


\title{
VUV spectroscopic investigation of HTSC and C60 materials
}

\author{
V.G. Stankevitch \\ Russian Research Center "Kurchatov Institute", 123182 Moscow, Russia
}

\begin{abstract}
Experimental confirmation of the existence of intrinsic luminescence in HTSCs and solid C60 is presented. Models of luminescence centers, including the self-trapped excitons formation, are proposed. We conclude, that luminescence methods are fruitful and helpful to obtain additional information on the electronic structure and its excited states.
\end{abstract}

\section{Introduction}

The present work is devoted to employing luminescence methods for investigations of the new superconducting materials, such as HTSCs and organic superconductors based on Carbon-60. It is well known that luminescence probe methods are widely used for material analysis, impurity testing, etc. However, an intrinsic luminescence, if there is any, may give new valuable information on the creation, and decay of electronic excitations in solid state, thus complementing the standard methods of electronic structure investigations.

\section{High temperature superconductors}

The present theoretical debates on HTSCs are focused on the problem of the electronic structure of the normal and superconducting states, and there is a large number of publications $[1,2]$, in which conventional methods of electronic metal structure studies were used for this purpose.

Here we present another way to understand the electronic structure of HTSCs, based on luminescence methods. Luminescence investigations, commonly used for insulators, seem to be quite untypical for metals. We explain the possibility to extend luminescence methods to HTSC problems due to 
large differences between the HTSC electronic structure and that of common metals. We also take advantage of the fact that HTSCs possess a metal-insulator structure where correlation effects play an important role.

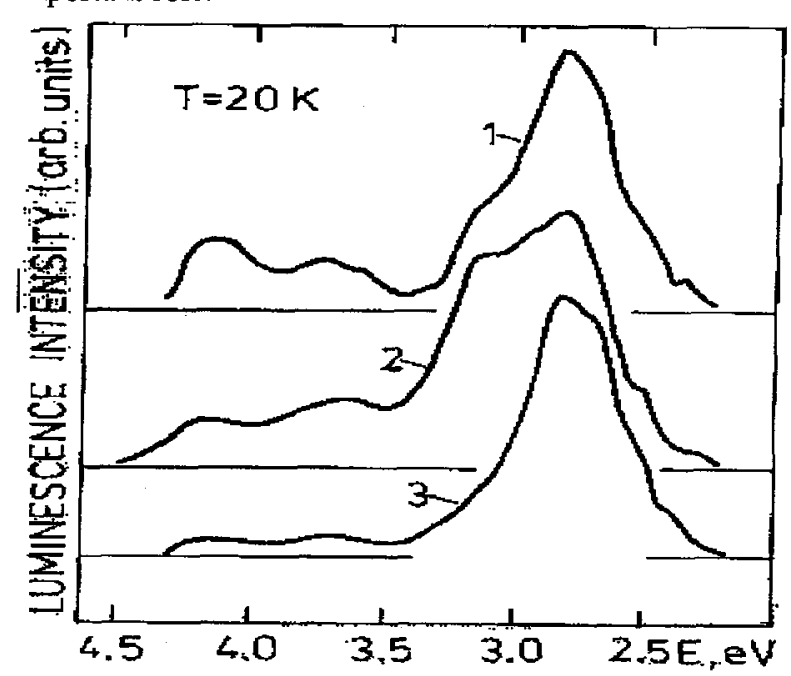

Fig. 1 Luminescence spectra of various single crystals after cleaving in situ, under excitation photon energy of $30 \mathrm{eV}$
The HTSCs have a complicated stoichiometry, and so, the procedure of crystal growth plays an important role, especially, for luminescence measurements. We tried to use the most perfect samples, prepared by different techniques, to eliminate the influence of random impurities. The most advanced investigations were done with $\mathrm{YBaCuO}$ cuprates [3-5].

Fig. 1 presents luminescence spectra of $\mathrm{YBa}_{2} \mathrm{Cu}_{3} \mathrm{O}_{7-\mathrm{x}}$ single crystals cleaved in ultrahigh vacuum at $\mathrm{T}=20 \mathrm{~K}$ immediately before the measurements, in order to exclude a possible influence on luminescent properties by chemical reaction products on the surface. Curve 1 corresponds to the crystal growing technique of Ref. [5], curves 2 and 3 correspond to another crystal growing procedure. Crystals on curve 2 were cleaved in the ab plane, while crystals on curve 3 were cut perpendicular to the $a b$ plane. However, the difficulty in obtaining a flat surface containing the $\mathrm{c}$ axis, as well as the geometry of observations, did not allow us to confirm that curves 2 and 3 are representative of accurate polarization measurements.

As one may see from Fig.1, all three emission spectra are similar, in first approximation, and correspond to earlier results for thin films [3]. Our luminescence spectra differ from the emission spectra of impurities, that could ever be produced in the process of crystal growth. The main contribution to emission gives a wide band peaked at $2.85 \mathrm{eV}$.

The nature of the less intensive $4.0 \mathrm{eV}$ luminescent band was not well determined in our experiments. Note, however, that it had a different temperature dependence, as well as luminescence quenching dependence under irradiation dose, than the main $2.85 \mathrm{eV}$ peak.

Luminescence quantum yield reached a value of $2 \%$ at $30 \mathrm{eV}$ excitation, the spectra shape being almost independent on the excitation energy. Luminescence excitation spectra displayed the increase of quantum yield at $\mathrm{E}>7 \mathrm{eV}$, and they were in antiphase to the reflectivity spectra [4], which suggests a bulk nature of luminescence.

All facts mentioned above led us to a conclusion, that a $2.85 \mathrm{eV}$ band corresponds to an intrinsic luminescence of $\mathrm{YBa}_{2} \mathrm{Cu}_{3} \mathrm{O}_{7-\mathrm{x}}$, and not to luminescence of random impurities in the bulk of the crystal or on its surface.

Our further investigations were directed to study the nature of the luminescence center. In a number of experiments [3] we observed some weak fine structure of the main luminescence band, arising at temperatures below Tc, and disappearing after the HTSC transition to a normal state. The results are shown in Fig.2. The right picture shows the temperature dependence of the $2.85 \mathrm{eV}$ peak. One may see two peculiarities in the luminescence spectra. First, the luminescence intensity rises rapidly at the temperature $\mathrm{Tc}$ of the superconducting transition. Second, at $\mathrm{T}<\mathrm{Tc}$ the main luminescence band has a weak structure. 

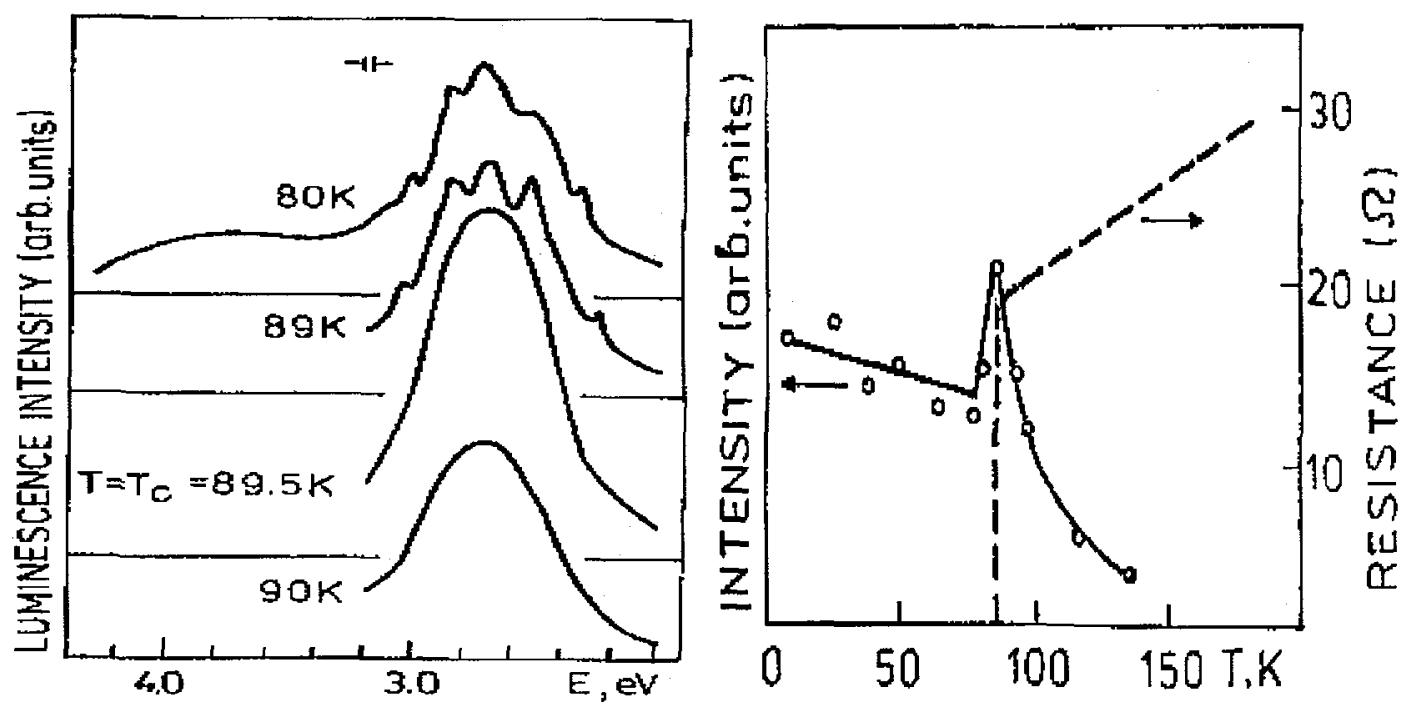

Fig. 2 Temperature dependences of the luminescenece spectra of $\mathrm{YBa}_{2} \mathrm{Cu}_{3} \mathrm{O}_{7-\mathrm{x}}$ film near $\mathrm{T}_{c}$ (left picture) and luminescence intensity (at $2.85 \mathrm{eV}$ ) with resistance measurements (right picture)

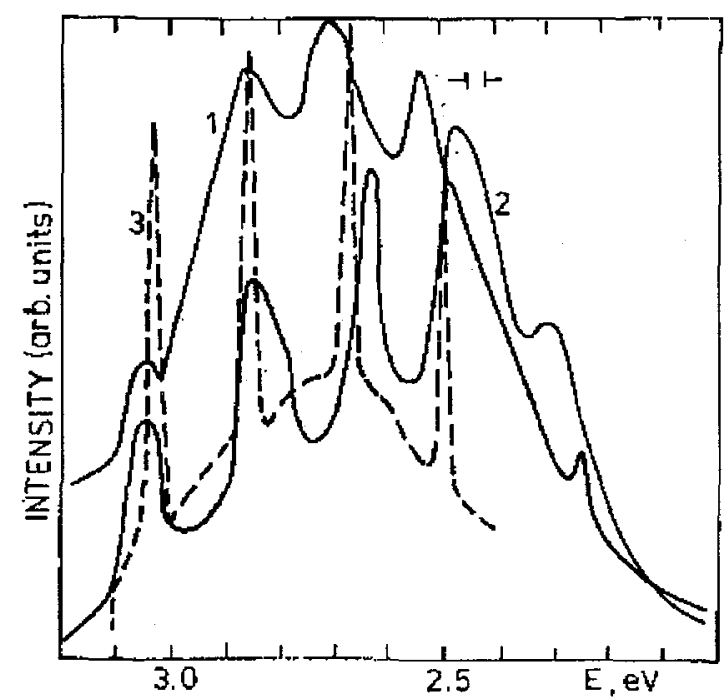

Fig.3 Spectra: (1) stationary luminescence of $\mathrm{YBa}_{2} \mathrm{Cu}_{3} \mathrm{O}_{7-\mathrm{x}}$ at $80 \mathrm{~K}$, (2) - laser luminescence of the same film, (3) oxygen luminescence in an $\mathrm{Ar}$ matrix at $6 \mathrm{~K}$.
It seems natural to interpret the fine structure as being due to vibrational transitions in the luminescence centers. This structure appeared to be more pronounced in time-resolved spectra obtained under pulsed laser excitation. In this case, it was possible to measure luminescence only in the time gate $100 \mu \mathrm{s}-1 \mathrm{~ms}$, due to a very low quantum yield at $4 \mathrm{eV}$ laser excitation energy (see curve 2 in Fig.3). For comparison, also shown are the emission spectra of molecular oxygen in argon matrix at $\mathrm{LHeT}$ (curve 3) and the spectrum of stationary luminescence of $\mathrm{YBa}_{2} \mathrm{Cu}_{3} \mathrm{O}_{7}$ film (curve 1), excited by $11.2 \mathrm{eV}$ photons. This vibrational spectrum corresponds to the partially forbidden transition ${ }^{3} \Delta_{\mathrm{u}} \quad \rightarrow^{3} \Sigma_{\mathrm{g}}^{-}$in the oxygen molecule, with a slow emission decay time $\sim 100 \mu$ s. Of course, a specific environment of the forming oxygen quasimolecule may partially reduce the degree of prohibition, thus decreasing the decay time. However, this may still remain sufficiently large.

The luminescence time-resolved measurements confirmed this suggestion. Luminescence appeared to display a weak ( $20 \%$ of total intensity) fast component of the nanosecond duration, while the main part of intensity ( $80 \%$ ) decays with a slow, $>5 \mu \mathrm{s}$, duration.

The vibrational quanta values in Fig. 3 for an oxygen and a $\mathrm{YBaCuO}$ emission seem to be rather similar $(0.17 \mathrm{eV})$. This leads to the conclusion that the luminescence centers in $\mathrm{YBaCuO}$ are also connected with molecular oxygen. 
Another confirmation of the important role of oxygen on the process of luminescence center formation was obtained in our experiments on radiation stability of HTSCs. We observed a strong quenching of luminescence (up to 10 times), even after irradiation by moderate doses of SR, of the order $10^{15}-10^{16} \mathrm{phot} / \mathrm{cm}^{2}$. Simultaneously, an oxygen molecular desorption was detected by means of a quadrupole mass-spectrometer.

If this assumption is true, the oxygen centers serve as luminescence probes. They are generated as a result of localization of electronic excitations, and then they decay radiatively or non-radiatively, including oxygen desorption. Unfortunately, it is difficult to determine a charge state and a structure of the quasimolecule that may include not only oxygen atoms, but also other atoms of the unit cell.

\section{Carbon-60}

The electronic structure of solid C-60 is a semi-conductor type, with an energy gap value scattering from $1.5 \mathrm{eV}$ to $2.4 \mathrm{eV}$ by different measurements [6]. Hence, the commonly observed wide photoluminescence band is situated in the region of optical transparency of the crystal.

Fig.4 [7] shows the absorption (curve 1) and luminescence spectra (curves 2 and 3) of thin solid

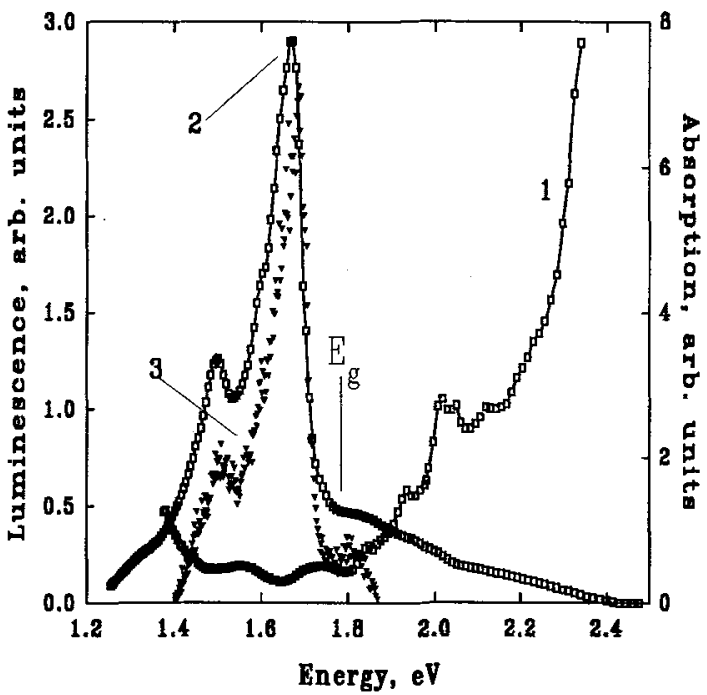

Fig 4. Absorption (1) and luminescence spectra under laser (2) and VUV undulator (3) photon beams. carbon film obtained, respectively, under laser $(E=3.68 \mathrm{eV}, \mathrm{T}=80 \mathrm{~K})$ and, for the first time, under VUV excitations $(\mathrm{E}=30 \mathrm{eV}, \mathrm{T}=10 \mathrm{~K})$. The luminescence spectra form a wide band, peaked at $\mathrm{E}=1.67 \mathrm{eV}$, with a Stock's shift value $\mathrm{E}-\mathrm{E}_{\mathrm{g}} \sim 0.2$ $\mathrm{eV}$. Besides, they have a weakly pronounced vibronic structure, including a small shoulder at $1.60 \mathrm{eV}$ and a peak at $1.50 \mathrm{eV}$. In addition, a high energy wide-band structureless emission was observed at $1.8-2.4 \mathrm{eV}$, its shape correlating with the absorption spectrum.

The temperature dependence of the integral luminescence intensity of solid C60 film is presented in a semi-logarithmic scale in Fig. 5 [7], for the temperature range $10-300 \mathrm{~K}$. The curve can be separated into three portions. For the first time, a luminescence jump was observed near the temperature of the structural phase transition $\mathrm{FCC}(\mathrm{Fm} 3) \rightarrow \mathrm{SC}(\mathrm{Pa} 3)$ at $\sim 250 \mathrm{~K}$ (see the inset in Fig.5). A step-like intensity increase on cooling may be attributed to this transition, accompanied by an abrupt orientational ordering reconstruction in solid $\mathrm{C} 60$ from isotropic molecular rotation of nearly spherical $\mathrm{C} 60$ molecules above $T_{c}$ to uniaxial jump reorientations around a single (111) direction below $T_{c}$ [8]. A corresponding abnupt decrease in the reorientational correlation time from $9.2^{*} 10^{-12} \mathrm{~s}$ to $2 * 10^{-9} \mathrm{~s}$ may lead to a probability decrease of electronic excitations relaxation through rotational modes. Such a behaviour of the luminescence intensity allows to conclude in favour of the intrinsic (bulk) nature of the luminescence in solid $\mathrm{C} 60$.

The main part of the spectrum, at $\mathrm{T}=13-250 \mathrm{~K}$ can be approximated by the well known relation assuming a pure temperature quenching of luminescence $[7,9]$ :

$$
I(t)=I_{0}\left(T_{0}\right) /\left\{1+\tau_{0} K_{n} \exp \left(-E_{a} / k T\right)\right\}
$$




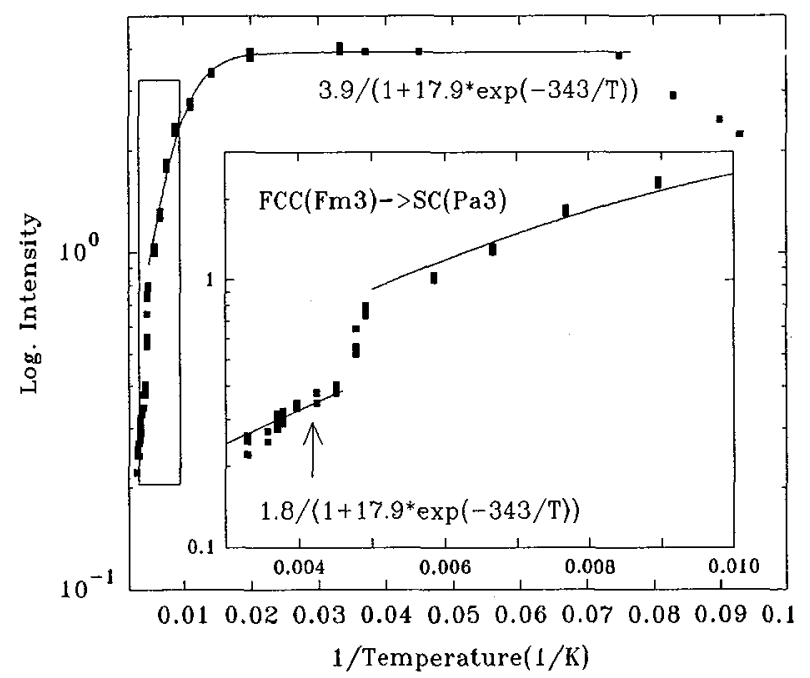

Fig. 5 Luminescence temperature dependence of solid C60 film under VUV undulator excitation
In this equation, $I_{0}, \tau_{0}$ are the intensity and the decay time at the low initial temperature $\mathrm{T}_{0} ; \mathrm{K}_{\mathrm{n}}$ is the rate constant for non-radiative relaxation of the first excited triplet state, responsible for emission; $E_{a}$ is the activation barrier between radiative and non-radiative transitions. The approximation of the experimental data by this formula gives the following parameter values: $\tau_{0} \mathrm{~K}_{\mathrm{n}}=17.9, \mathrm{E}_{\mathrm{a}}$ $=343 \mathrm{~K}$.

The third portion of the temperature dependence curve at $\mathrm{T}<13 \mathrm{~K}$ demonstrates the intensity decrease on further cooling. A similar behaviour may be interpreted by the presence of an activation barrier for localization of electronic excitations, similar to a barrier for selftrapping of excitons.

Time-resolved experiments on the integral luminescence intensity decay, performed with the VUV undulator excitation $(E=30 \mathrm{eV})$ at $\mathrm{T}=13 \mathrm{~K}$ [7], exhibited the following magnitude for a decay time: $\tau_{0}=1.2 \pm 0.1 \mathrm{~ns}$. Probably, this indicates a singlet nature of this state. The absence of a more prolonged time component may be attributed to a non-radiative relaxation of the first excited triplet state $T_{1}$. The rate constant for intercombinational transition $S_{1} \rightarrow T_{1}$ corresponds to that of non-radiative relaxation $K_{n}$. Taking the experimental value for $\tau_{0}$ and parameters from equation (1), one can deduce the value $K_{n}=1.5^{*} 10^{10} \mathrm{~s}^{-1}$, which is close by the order of magnitude to the value $K_{n}=3^{*} 10^{10} s^{-1}$ for free C60 molecules [10].

Thus, in relaxation process the key role is plaid by $\mathrm{C} 60$ clusters, forming the crystal. Apparently, a non-radiative relaxation of excited states in solid $\mathrm{C} 60$ film leads to certain structural deformations followed by a dose degradation of luminescence intensity, observed under laser [11] and VUV [7] excitations. As it was noticed in Ref. [12], a photo-dimerization can occur between one monomer in the first excited triplet state and the second monomer in the ground state, leading to the covalent bonding of $\mathrm{C} 60$ molecules. This mechanism may, possibly, reduce the number of $\pi$-bonds responsible for radiative transitions in our case.

The results of luminescence experiments on solid film "in situ" doping by potassium atomic beam are reported in Ref.[7, 11]. These systems gave the following results: 1) The doping procedure lead to a proportional emission intensity decrease, observed on doping at $400 \mathrm{~K}$. The cooling of doped samples increased the luminescence, however, less than for pure C60.2) The decrease rate of emission in doping was lower, than the growth rate of concentration of the free carriers in bulk doped C60. This fact may be evidence that the partial luminescence quenching in doped samples is not due to free carriers, but to formation of the additional quenching centers, like $\mathrm{K}_{3} \mathrm{C}_{60}$.

Therefore, the experimental data allow to conclude that the observed emission is intrinsic, because its intensity correlates with the changes of bulk properties. The wide luminescence band, the Stock's shift, the partial quenching of emission by doped potassium atoms, dose and temperature degradation of luminescence at $\mathrm{T}<13 \mathrm{~K}$, as well as the nanosecond decay time, indicate to its connection to self-trapped excitons.

As a result, the following model of luminescence centers in solid $\mathrm{C} 60$ is proposed. The electronic excitations of excitonic type are localized on the two-dimensional frame of the C60 molecule, preferably, near the relatively weak $\pi$-bonds, connecting the $\mathrm{C}$-atom hexagons in the molecule. As a result of structural deformation, a $\mathrm{C}_{2}^{*}$ quasi-molecule is formed (the so called process of self-trapping), that exists only in the excited state, and may decay radiatively or non-radiatively. 
In summary, we suggest that both HTSC and $\mathrm{C} 60$ possess the similar mechanisms of localization of electronic excitations with formation of luminescence centers.

I thank all my colleagues who participated in these investigations and helped to prepare this work.

\section{References}

1. A.Samsavar et al., Phys.Rev.B37 (1988) 5164.

2. A.J.Viescas et al., Phys.Rev.B37 (1988) 3738.

3. V.G.Stankevitch, N.Yu.Svechnikov, K.V.Kaznacheev, et al, NIM A282 (1989) 684-687.

4. V.G.Stankevitch, N.Yu.Svechnikov, K.V.Kaznacheev, et al., Journ. Lum. 48\&49 (1991) 845-848.

5 V.G.Stankevitch, N.Yu.Svechnikov, K.V.Kaznacheev, et al., Phys.Rev. B47 No.2 (1993) 1024-1028.

6. T.Rabenan, A.Simon, R.K.Kremer, E.Sohman, Z.Phys.B90 (1993) 69.

7. M.A.Terekhin, N.Yu.Svechnikov, V.G.Stankevitch, A.A.Kolmakov, V.A.Stepanov, V.N.Bezmelnitzin, M.Kamada, K.Kan'no, Journ. Appl. Spectroscopy (to be published). In Russian.

8. David W.I.F., Ibberson R.M., Dennis T.J.S., Hare J.P., Prassides K., Europhys. Letts. 18 (1992) 219.

9. V.V.Antonov-Romanovskii, Kinetics of crystallo-phosphors' luminescence (Edit. by "Nauka", Moscow, 1966), p.324. In Russian.

10. M.R.Wasilewski, M.P.O'Noil, K.R.Lykke, M.J.Pellin, D.M.Cruen, Journ. Amer. Chem. Soc. 1B (1991) 2774.

11. A.A.Kolmakov, V.G.Stankevitch, N.Yu.Svechnikov, M.A.Terekhin, K.V.Kaznacheev, Photoinduced properties of solid buckminsterfullerene films, Preprint IAE-5644/9, Russian Research Center "Kurchatov Institute", Moscow, 1993.

12. Y.Wang, J.M.Holden, Z.-H.Dong, X.-X Bi, P.C.Eklund, Chem. Phys. Letts. 211 (1993) 341. 